\title{
Plasmodium yoelii: Identification of a Gene Encoding a Putative ADP-ribosylation Factor-1 GTPase-activating Protein, PyAG1
}

\author{
Rémi Hienne $^{+}$, Alain Rico, Daniel Parzy, Jean-Claude Doury \\ Unité de Parasitologie, Institut de Médecine Tropicale du Service de Santé des Armées, Parc du Pharo, BP 46, \\ 13998 Marseille Armées, France
}

The PyAG1 gene, identified by the screening of a Plasmodium yoelii genomic DNA library with a rhoptry-specific Mab, encodes a protein with a zinc finger structure immediately followed by the consensus sequence of the Arf GAP catalytic site. The serum of mice immunized with the recombinant protein recognized specifically the rhoptries of the late infected erythrocytic stages. Blast analysis using the Genbank database gave the highest scores with four proteins presenting an Arfl GAP activity. If presenting also this activity, the PyAG1 protein could be involved in the regulation of the secreted protein vesicular transport and, consequently, in the rhoptry biogenesis.

Key words: Plasmodium yoelii - gene PyAG1 - immature rhoptries - vesicular transport

Rhoptries are located at the apical end of the invasive stages of all Apicomplexan parasites and have morphological characteristics of secretion organelles. Their contents are secreted into the host cell during invasion and play a critical role in the invasion process (Sam-Yellowe 1996). It has been proposed that rhoptries are formed de novo at the end of the asexual erythrocytic cycle by budding of secretory vesicles from the Golgi apparatus (GA), a process analogous to the secretory granules of mammalian cells (Porchet \& Torpier 1977). Therefore, the rhoptry components must be synthesized every cycle and transported to the organelles via a secretory pathway involving the GA. The fact that this transport (Ogun \& Holder 1994, Howard \& Schmidt 1995) as well as the rhoptry maturation (Ward et al. 1997) can be blocked by brefeldin A (BFA) might indicate the intervention of coated vesicles the formation of which is regulated by the GTP-binding ADP-ribosylation factor (Arf) cycle (Becker \& Melkonian 1996). In-

\footnotetext{
This work is supported by grants from GDR "CNRSDGA/DSP".

${ }^{+}$Corresponding author. Fax: +33-4-9159.4477. E-mail: imtssa@gulliver.fr

Received 21 May 1999

Accepted 27 January 2000
}

deed, this fungal toxin maintains the Arf protein in an inactive form (Arf-GDP) by preventing the GDP/GTP exchange. This data and the microscopic observation of coated vesicles during apical organelles maturation (Bannister \& Mitchell 1995) could imply that the GTP-Arf cycle plays an important role in the rhoptry biogenesis.

This short communication reports the cloning of a novel Plasmodium yoelii gene encoding a putative Arf1 GAP (GTPase-activating protein) which seems to be associated with the immature roptries of the 4-8 nucleus schizonts.

An EcoRI library of $P$. yoelii genomic DNA, in $\lambda$ ExCell EcoRI/CIP (Amersham Pharmacia Biotech), was screened with a monoclonal antibody (Mab), named C5-10. This Mab belongs to a Mab library which specifically reacts, by immunofluorescence assay (IFA), with the $P$. yoelii rhoptries (Fig. 1A); an immunoelectron microscopy analysis confirmed this localization (Hienne et al. 1998). By immunoblot under reducing conditions (IB/R), the C5-10 Mab recognizes a major protein of 68 $\mathrm{kDa}$ and a minor doublet of $31 / 34 \mathrm{kDa}$ (Fig. 2A).

From the genomic DNA library, a recombinant lambda phage, $\lambda$ AT711, containing a 1011bp insert, was isolated. This DNA insert presented an open reading frame of 885-bp but no initiation codon. To obtain the full-length sequence at the 5' end of the gene, we performed inverse PCR with three combinations of six oligonucleotides (C1, C2, C3, D1, D2, D3), using P. yoelii genomic DNA HindIII digests (Fig. 3). 

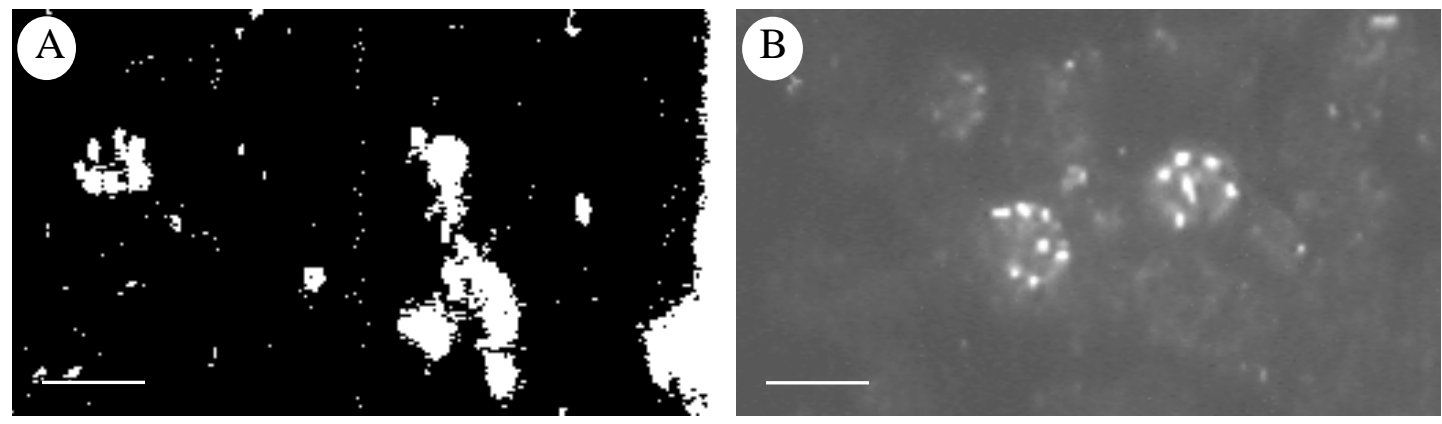

Fig. 1: immunofluorescence on air-dried erythrocytes parasitized by late asexual blood stage of Plasmodium yoelii. A: with the C5$10 \mathrm{Mab}$; B: with the serum of a female BALB/c mouse (Charles River) immunized against the GST-PyAG1 recombinant protein. Bar $=10 \mu \mathrm{m}$.

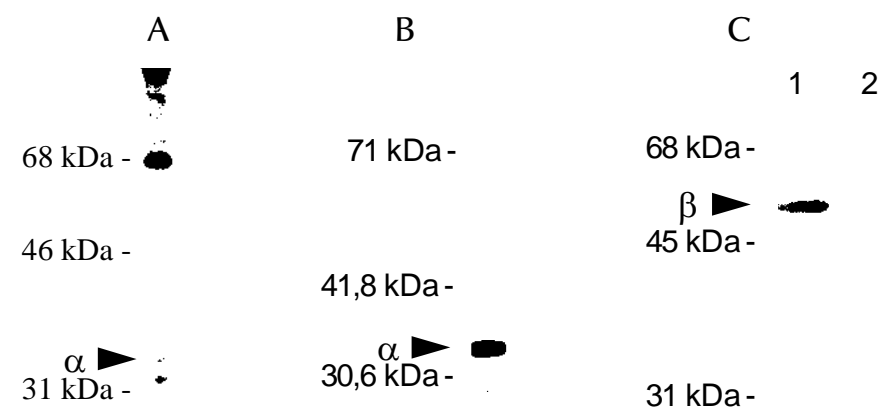

Fig. 2A, B: immunoblots of whole schizont extracts of Plasmodium yoelii, prepared under reducing conditions and incubated with (A) the C5-10 Mab or (B) the serum of a female BALB/c mouse (Charles River) immunized against the GST-PyAG1 recombinant protein; C: immunoblot of the GST-PyAG1 recombinant protein (lane 1) or the native GST protein (lane 2), prepared in reducing conditions and incubated with the C5-10 Mab. The development is realized with horseradish peroxidase-conjugated goat antimouse $\mathrm{IgG}(\mathrm{H}+\mathrm{L})$ (Jakson) and the ECL ${ }^{\mathrm{TM}}$ Western blotting detection reagents (Amersham). The interesting bands positions are marked by arrows - $\alpha$ : PyAG1 $\left(\mathrm{MW}_{\text {app }} \cong 34 \mathrm{kDa}\right) ; \beta$ : GST-PyAG1 recombinant protein $\left(\mathrm{MW}_{\text {app }} \cong 58 \mathrm{kDa}\right)$.

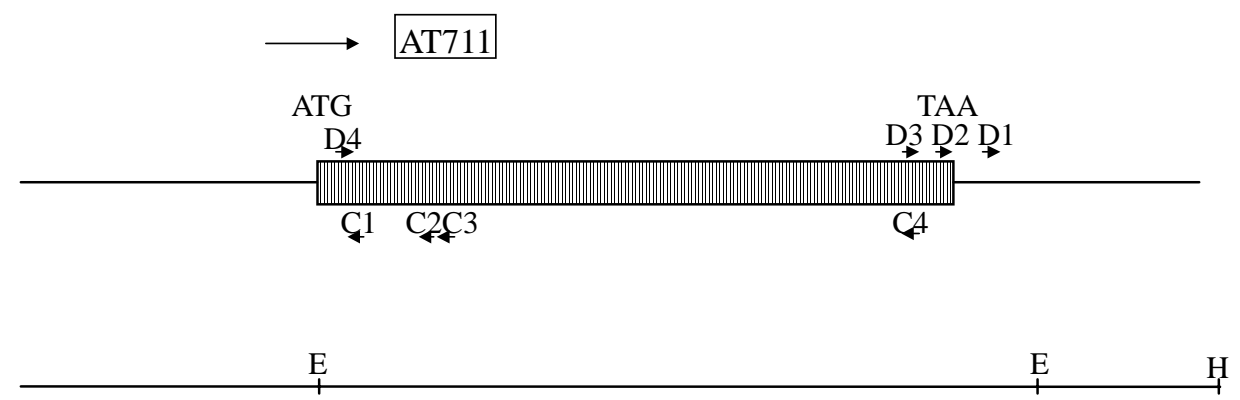

$\underline{100 \mathrm{bp}}$

Fig. 3: schematic presentation of the gene encoding putative zinc finger protein AT711. The open reading frame is represented by the shaded open box. The primers position is indicated by arrows: $\mathrm{C1}_{65-43}\left(5^{\prime}-\mathrm{CAT}\right.$ TTA TTA TTA CTT TCA TCG TC-3'); $\mathrm{C}_{161}{ }_{16}$ 141 (5'-ACC CCC AAA CTT CTA TGA ACC-3'); C3 ${ }_{192-168}$ (5'-CAT TTT TAT ACT TCT TAC TAC ACT T-3'); C4 ${ }_{843-819}\left(5^{\prime}-\right.$ TGG TTT TGA CTC GTT GTT ATT GTT C-3'); D1 ${ }_{933-957}$ (5'-ACC TCG GAA TGC AAA TAT AA-3'); D2 $268-890$ (5'-GGA AAT GGT ACA AAT GAA GCA TA-3'); D3 ${ }_{821-843}$ (5'-ACA ATA ACA ACG AGT CAA AAC CA-3'); D4 ${ }_{27-51}$ (5'-TAC CAA ATT AAA AAA AGA CGA TGA A-3'). The restriction sites used in the cloning strategy and in the restricted PCR are marked: E, EcoR I; H, Hind III. 
Two nested amplifications were carried out with the oligonucleotide pairs C3/D3 and C2/D2. The sequence, obtained with the oligonucleotide $\mathrm{C} 1$, contained a methionine codon as well as upstream stop codons in frame with the putative initiation codon. The complete nucleotide and derived amino acid sequences (Fig. 4) of this novel gene, named PyAG1, are available in the GenBank ${ }^{\mathrm{TM}}$ data base under the accession number AF055920. To confirm the synthesis of this putative protein during Plasmodium erythrocytic cycle, we isolated poly $(\mathrm{A})^{+} \mathrm{RNA}$ from late asexual stages of $P$. yoelii with Dynabeads kit (Dynal), after DNase treatment of the total RNA solution, and carried out RT PCR. The PCRamplification and sequencing of cDNA, using the two oligonucleotides C4 and D4 (Fig. 3), demonstrated that PyAG1 gene is transcribed (data not shown).

This gene has an open reading frame of 888bp in length which encodes a hydrophilic protein of 296 amino acids ( $33 \mathrm{kDa}$ ). This protein presents, at its N-terminus, two interesting motifs: a zinc finger element (spanning residues 22-45) of the form $\left[\mathrm{C}-(\mathrm{X})_{2}-\mathrm{C}-(\mathrm{X})_{16}-\mathrm{C}-(\mathrm{X})_{2}-\mathrm{C}\right]$ (with $\mathrm{C}$, cysteine; $\mathrm{X}$, any amino acid) immediately followed by the consensus sequence of the Arf GAP catalytic site (Scheffzek et al. 1998) (spanning residues 47-53) of the form [s-h-H-R-x-h-x] (with s, glycine or alanine; h, hydrophobic amino acid; $\mathrm{H}$, histidine; $\mathrm{R}$, arginine; $x$, any amino acid).

A phylogenetic analysis by sequencing with the D4 and C3 oligonucleotides, using genomic DNA of rodent ( $P$. yoelii nigeriensis, $P$. berghei, $P$. chabaudi adami, $P$. vinckei petteri) and human $(P$. falciparum Palo Alto and 3D7) plasmodial species, revealed an important preservation of this interesting region (Fig. 5, Table IA). This observation was confirmed by the sequencing of the 285 first nucleotides of the $P$. falciparum homologous gene (Fig. 5 and Table IB).

The PyAG1 gene product, expressed as glutathione S-transferase fusion protein (GSTPyAG1) in Escherichia coli (pGEX-3X plasmid/ GST Gene Fusion System, Amersham Pharmacia Biotech), was recognized by the Acm C5-10, using IB/R (Fig. 2C). By IFA, the serum of female BALB/c mice (Charles River, France), immunized with the recombinant protein, recognized specifically red blood cells infected by $P$. yoelii young schizonts (4-8 nuclei), with a rhoptry-like labelling pattern (Fig. 1B). An immunoelectron microscopy study will be required to confirm this ultrastructural localization. By IB/R, this polyclonal antibody confirmed the presence of the PyAG1 gene product in a reduced antigenic extract of $P$. yoelii mature erythrocytic stages (Fig. 2B).

Blast analysis using the Genbank ${ }^{\mathrm{TM}}$ database gave the highest homology scores with four proteins presenting the same two interesting motifs in a similar position and an Arf1 GAP activity: Arf1 GAP of Arabidopsis thaliana (Genbank accession number AC004684), Drosophila melanogaster (Genbank accession number AF011427), Rattus norvegicus (Cukierman et al. 1995), and Gcs1 of Saccharomyces cerevisiae (Ireland et al. 1994, Poon et al. 1996) (Fig. 6, Table II). The structural homology with these proteins and the presence of the consensus sequence of the Arf GAPs catalytic site allowed us to hypothetize that the PyAG1 gene product may possess an Arf1 GAP activity. This activity steps in the Arf-GTP cycle by catalysing the GTP hydrolysis and, consequently, the transport vesicle uncoating, indispensable step for the membrane fusion between the vesicles and the target membrane.

The specific labelling of the immature rhoptries with polyclonal anti-PyAG1 serum corroborates this putative activity. Indeed, at first schizont stages, the parasites present immature rhoptries with low density $\left(1.12 \mathrm{~g} . \mathrm{ml}^{-1}\right)$ on sucrose gradient, even though the rhoptries have a significantly greater density in sucrose $\left(1.16 \mathrm{~g} \cdot \mathrm{ml}^{-1}\right)$ at the mature schizonts, consequence of the accumulation of rhoptry proteins probably transported through coated vesicles (Jaikaria et al. 1993).

Therefore, the PyAG1 protein may interfere with the regulation of the secreted proteins vesicular transport and, consequently, with the biogenesis of the secreting organelles like rhoptries. The identification of such an activity supports the presence of a classical eukaryotic transport pathway involving coated vesicules in malarial parasite which has been suggested by BFA-inhibition experiences (Crary \& Haldar 1992, Benting et al. 1994, Das et al. 1994, Hinterberg et al. 1994, Ogun $\&$ Holder 1995, Howard \& Schmidt 1995) and $P$. falciparum Arf or Arl (ADP-ribosylation factorlike) characterization (Stafford et al. 1996, Lee et al. 1997, Truong et al. 1997).

Through this preliminary study, we have identified a new element of the intracellular protein transport between plasmodial organelles. Due to its putative regulator activity on the secreting organelles biogenesis, this protein could become a new target with a view to inhibit the parasite development.

\section{ACKNOWLEDGEMENTS}

To Drs P Falanga, Y Boulard, and Prof. I Landau for the providing of parasite strains and species. 
tttttttctcctttatatatacttttttaagaattttatttttatgtaaacgatatttttttcattatatatatttaattattaaatctttctttgtattatatatacaattgtgcatataatattca cacgtttcttttcttcttattattattattattcccatatttatagtagtctttgcgttttgggaaaaattcaattttaagagtcatgcatcatcactgatttattataatatatataattatat tattaacccgtatgaatttgataaagatattttatataattaatatctttacaaaatatacataccgatttgaatattttttgtacacacatatatattatacaaaccctataactttacagtt

$\begin{array}{llllllllllllllllllll}D & E & S & N & N & K & C & F & D & C & G & I & P & N & P & D & W & V & S & V\end{array}$

GCA TAA aataataatatattaaaattgttaataatcatagtaataaaacctcggaatgcaaatataagaagtaaattccccataaaattagaatcgaaatacttcacaaaattt

Fig. 4: nucleotide and predicted amino acid sequences of the gene encoding the putative zinc finger protein. Inserts obtained from $\lambda$ Excell-positive plaques or from inverted PCR experiments were sequenced in both orientations. DNA sequencing was carried out by cycle sequencing, using dye terminators on an ABI model 310 automated DNA sequencer (Applied Biosystems). The putative zinc finger domain is underlined and the consensus sequence of the Arf GAP catalytic site is double-underlined. The nucleotides and amino acids are numbered on the right. Lower-case letters indicate non-coding regions and $(*)$ the in-frame stop codon. 


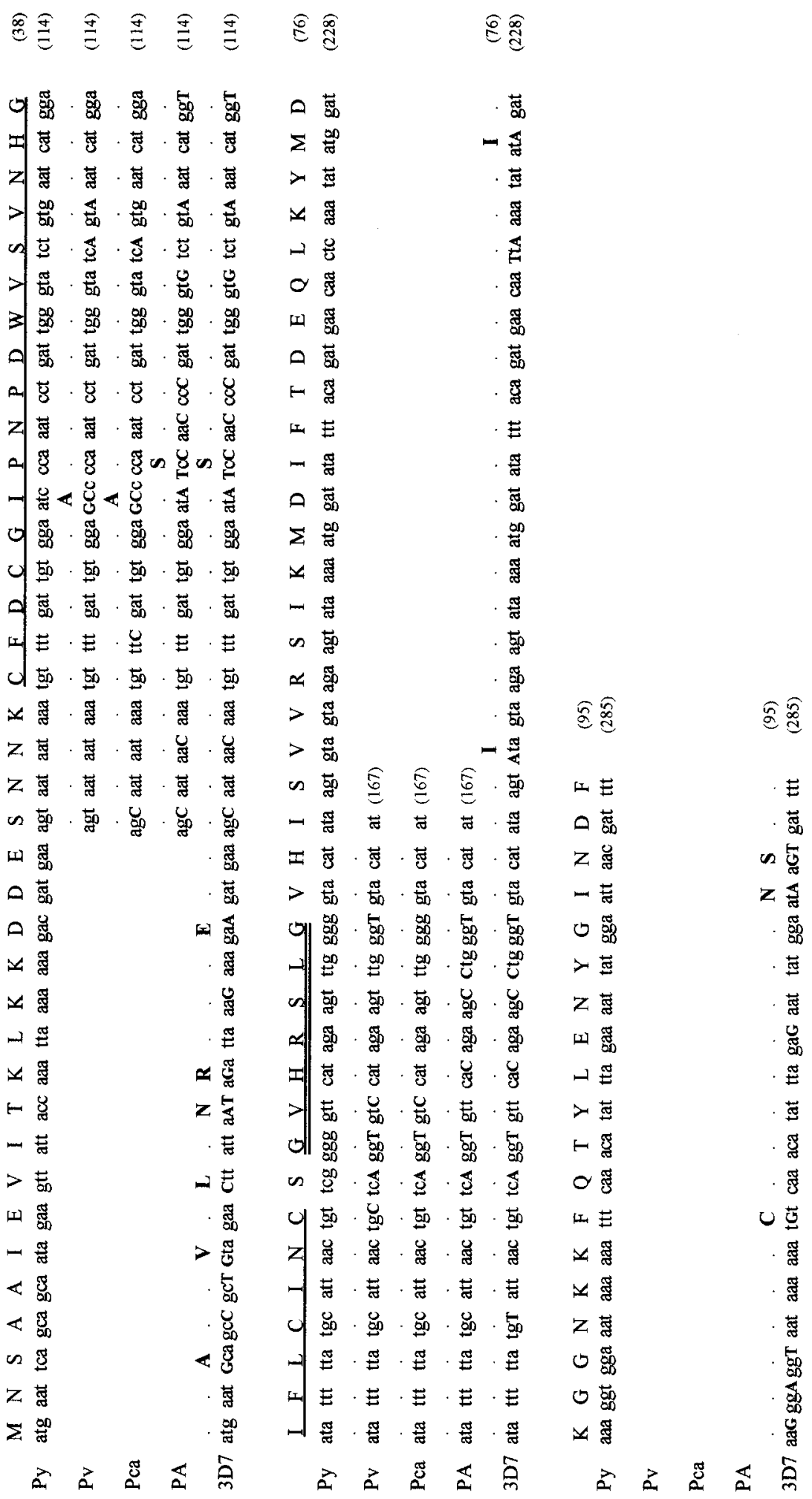

Fig. 5: preservation of the PyAG1 nucleotide and predicted amino acid sequences through different malaria species. Py: Plasmodium yoelii; Pv: P. vinckei petteri 279BY; Pca: P. chabaudi adami 887KA; PA: P. falciparum Palo Alto; 3D7: P. falciparum 3D7. Different nucleotides or amino acid are in capital bold types. Points indicate identical amino acids. The putative zinc finger domain is underlined and the consensus sequence of the Arf GAP catalytic site is double-underlined. The nucleotides and amino acids are numbered on the right. 


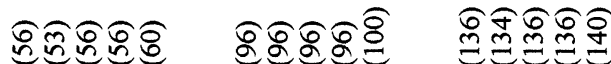

\begin{tabular}{|c|c|c|c|}
\hline & & & \\
\hline ロルヘルロ & ローカッカ & $\mathbf{Z}-\mathbf{Z}=-$ & \\
\hline 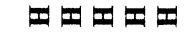 & $4-0=\infty$ & - 1 on $n$ & \\
\hline$\vec{p}>>>$ & $\ominus^{\ominus}$ Z几ー & -1000030 & \\
\hline 비비비비 & Z的 $3>0$ & I $00>$ Z & \\
\hline - & - & $\not>\simeq \sigma \sigma$ & \\
\hline se of 20 on on & Z ס ט Z & ט & \\
\hline 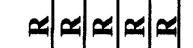 & $\nu \nu \sigma \sigma I$ & 뵈 되 & \\
\hline$\pm \pm= \pm$ & $\mathrm{Z} \sigma \theta \approx \sim$ & $0 \not \infty \infty ⿻$ & \\
\hline$>-\because=-1$ & 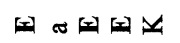 & $\mu>0 n$ & \\
\hline பு & دـ د د د & $2 .-\not 100$ & \\
\hline $\cos \cos \ll$ & 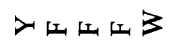 & ـ ـ ג & \\
\hline$\rightarrow \cup \cup \cup \cup \cup$ & $H=000$ & $A \sim O$ in 0 & \\
\hline $\begin{array}{lllll}Z & 0 & 0 & 0 & 0\end{array}$ & $\sigma=\alpha \approx+$ & 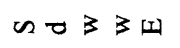 & \\
\hline ールヘル & $x_{1}-\infty x_{1}$ & $2+$ in 0 w & \\
\hline טن ט ט & $\forall \propto ⿻ \mathscr{L}$ & $23 y y>$ & \\
\hline 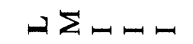 & $\forall$ 山 几 & $Z \sigma 0000-$ & \\
\hline 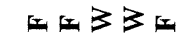 & $\mathbf{Z Z Z Z Z Z ~}$ & 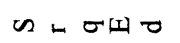 & \\
\hline ロールール & ט ט ט ט & $=00 \pi \approx 0$ & \\
\hline ט ט ט ט ט & ט ט ט ט ט & $z_{0} 0-0$ & \\
\hline Iメンメ & $\mathbb{1} \approx \pi \mathbb{A}$ & $>\ll+\omega \omega$ & \\
\hline Z必 & 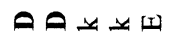 & ロ川 & \\
\hline$>->>a$ & $\Sigma \Sigma \Sigma \Sigma \Sigma$ & $n<. .>H$ & \\
\hline $\operatorname{nos} n t$ & $\lambda \not x \not y-$ & 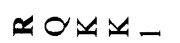 & \\
\hline$><>><$ & 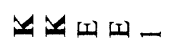 & ע & \\
\hline$B B B Z B$ & ఎ ఎ ح &.$-\omega t 0$ & \\
\hline$\sigma \sigma \sigma \sigma \sigma$ & சன山ய山 & $\sigma \nabla>4$ & \\
\hline$A \sim \approx A \infty$ & 뵈……되 & $\simeq \simeq-\infty$ & \\
\hline $\mathbf{z} \mathbf{z} \mathbf{z} \mathbf{z}$ & $A$ MA口 & $\lambda \nu \pi \approx 0$ & 앙ㅇㅇㅇㅇㅇ \\
\hline$A \not A$ A & 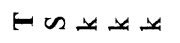 & 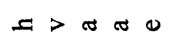 & $\Xi \Xi \Xi \Xi こ$ \\
\hline$-\sigma+\sigma \sigma$ & WBBa & 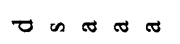 & \\
\hline ט & $-\infty \not \forall \forall \sigma$ & 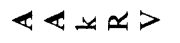 & $\infty$ os $Z \varangle \ll$ \\
\hline$\rightarrow \cup \cup \cup \cup \cup$ & คคคคด & $\ll<\infty n n$ & $\lambda \sigma n+\infty$ \\
\hline คの山ロ & $\Sigma \Sigma \Sigma \Sigma \Sigma$ & $\forall=\approx n a$ & 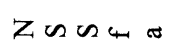 \\
\hline$E>E E$ & $\not{y}+++$ & $\operatorname{rn} \lambda>0$ & $>\mu$ on $\sigma \ll$ \\
\hline$\rightarrow \cup \cup \cup \cup \cup$ & $->>>-$ & 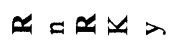 & $Z=\infty$ \\
\hline 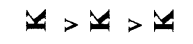 & $\operatorname{nos} n \cos$ & $\lambda \nabla \sigma \not$ & $\mu$ en $-O$ \\
\hline $\mathbf{Z} \leadsto \boldsymbol{Z} \mathbf{Z}$ & $\simeq \simeq \simeq \simeq \simeq$ & $\forall \approx+\sigma>$ & $\mathrm{Z} x \sim \simeq \infty$ \\
\hline $\mathbf{z} \mathbf{z} \mathbf{z} \mathbf{z}$ & $>>>>$ & $\simeq \infty . . \forall$ & $-\not x+\infty$ \\
\hline$\infty 000 \pi$ & $>4+44$ & II $\operatorname{enc} \theta$ & $v \approx \infty \sigma \infty$ \\
\hline I I & 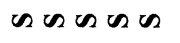 & $A, \approx \beta A$ & $>E \equiv 2 \infty$ \\
\hline
\end{tabular}

\begin{tabular}{|c|c|c|}
\hline 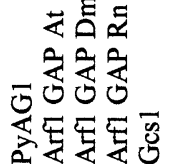 & 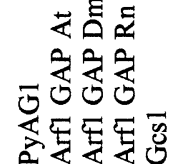 & 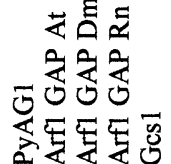 \\
\hline
\end{tabular}

Fig. 6: the $\mathrm{NH}_{2}$-terminal sequences of PyAG1, ARF1-GAP of Rattus norvegicus and Drosophila melanogaster, Gcs1 of Saccharomyces cerevisiae are compared with the Blast program. Amino acid identities and similarities are in capital bold types and in capital types, respectively. The position of the four cysteines forming the zinc finger motif is marked by arrows. The consensus sequence of the Arf GAP catalytic site is underlined. Residue numbers are shown on the right. 


\section{TABLE I}

Preservation of the PyAG1 nucleotide and predicted amino acid sequences through different malaria species. A: variability of the nucleotide sequence [52-167] amplified with the oligonucleotide pair D4/C3; B: variability of the 285 first nucleotides of the Plasmodium falciparum 3D7 homologous gene. The following groups of amino acids were designated as similar: $[\mathrm{K}, \mathrm{R}],[\mathrm{M}, \mathrm{V}, \mathrm{L}, \mathrm{I}, \mathrm{F}],[\mathrm{F}, \mathrm{Y}, \mathrm{W}],[\mathrm{S}, \mathrm{T}],[\mathrm{E}, \mathrm{D}],[\mathrm{N}, \mathrm{S}]$

\begin{tabular}{|c|c|c|c|}
\hline \multirow[t]{2}{*}{ A } & Nucleotides & \multicolumn{2}{|c|}{ Amino acids } \\
\hline & Identity (\%) & \multicolumn{2}{|c|}{ Identity and similarity (\%) } \\
\hline P. yoelii nigeriensis $798 \mathrm{VK}$ & 100 & \multicolumn{2}{|c|}{100} \\
\hline P. berghei NKK173 & 100 & \multicolumn{2}{|c|}{100} \\
\hline P. vinckei petteri $279 \mathrm{BY}$ & 94 & \multicolumn{2}{|c|}{97.4} \\
\hline P. chabaudi adami $887 \mathrm{KA}$ & 91.4 & \multicolumn{2}{|c|}{97.4} \\
\hline P. falciparum Palo Alto & 86.2 & \multicolumn{2}{|c|}{97.4} \\
\hline P. falciparum 3D7 & 85.3 & \multicolumn{2}{|c|}{97.4} \\
\hline \multirow[t]{2}{*}{$\mathrm{B}$} & Nucleotides & \multicolumn{2}{|c|}{ Amino acids } \\
\hline & Identity (\%) & Identity (\%) & Similarity $(\%)$ \\
\hline P. falciparum 3D7 & 86 & 87.4 & 94.7 \\
\hline
\end{tabular}

\section{TABLE II}

Homology between the deduced amino acid sequences of PyAG1 gene product and the Arf1 GAP of Arabidopsis thaliana (At), Drosophila melanogaster (Dm), Rattus norvegicus (Rn) and Saccharomyces cerevisiae (Gcs1)

Amino acids 17-121

(minimal sequence presenting an Arf GAP activity in $R$. norvegicus Arf1 GAP)

(Cukierman et al. 1995)

\begin{tabular}{|c|c|c|c|c|}
\hline & \multicolumn{2}{|c|}{ (Cukıerman et al. 1995) } & \multicolumn{2}{|c|}{ sequence) } \\
\hline & Identity (\%) & Similarity $(\%)$ & Identity (\%) & Similarity (\%) \\
\hline Arf1 GAP At & 45.7 & 63.8 & 56.3 & 75 \\
\hline Arf1 GAP Dm & 38.1 & 56.2 & 65.6 & 81.3 \\
\hline Arf1 GAP Rn & 38.1 & 57.1 & 62.5 & 78.1 \\
\hline Gcs1 & 37.1 & 58.1 & 53.1 & 71.9 \\
\hline
\end{tabular}

Amino acids 22-53

(sequence containing the zinc finger element and the Arf GAP consensus sequence) 
to cell proliferation. EMBO 13: 3812-3821.

Jaikaria NS, Rozario C, Ridley RG, Perkins ME 1993. Biogenesis of rhoptry organelles in Plasmodium falciparum. Mol Biochem Parasitol 57: 269-280.

Lee F-JS, Patton WA, Lin CY, Moss J, Vaughan M, Goldman ND, Syin C 1997. Identification and characterization of an ADP-ribosylation factor in Plasmodium falciparum. Mol Biochem Parasitol 87: 217 223.

Ogun SA, Holder AA 1994. Plasmodium yoelii: Brefeldin A-sensitive processing of proteins targeted to the rhoptries. Exp Parasitol 79: 270-278.

Poon PP, Wang X, Rotman M, Hubert I, Cukierman E, Cassel D, Singer RA, Johnston GC 1996. Saccharomyces cerevisiae Gcs1 is an ADP-ribosylation factor GTPase-activating protein. P Natl Acad Sci USA 93: 10074-10077.

Porchet E, Torpier G 1977. Etude du germe infectieux de Sarcocystis tenella et Toxoplasma gondii par la technique de cryodécapage. Zeitschrift für
Parasitendunke 54: 101-124.

Sam-Yellowe TY 1996. Rhoptry organelles of the apicomplexa: their role in host cell invasion and intracellular survival. Parasitol Today 12: 308-316.

Scheffzek K, Ahmadian MR, Wittinghofer A 1998. GTPase-activating proteins: helping hands to complement an active site. Trends Biochem Sci 23: 257-262.

Stafford WHL, Stockley RW, Ludbrook SB, Holder AA 1996. Isolation, expression and characterization of the gene for an ADP-ribosylation factor from the human malaria parasite, Plasmodium falciparum. Eur J Biochem 242: 104-113.

Truong RM, Francis SE, Chakrabarti D, Goldberg DE 1997. Cloning and characterization of Plasmodium falciparum ADP-ribosylation factor and factor-like genes. Mol Biochem Parasitol 84: 247-253.

Ward GE, Tilney LG, Langsley G 1997. Rab GTPases and the unusual secretory pathway of Plasmodium. Parasitol Today 13: 57-62. 